\title{
A Nonlinear Flux Approximation Scheme for the Viscous Burgers Equation
}

\author{
N. Kumar, J.H.M. ten Thije Boonkkamp, B. Koren and A. Linke
}

\begin{abstract}
We present a nonlinear flux approximation scheme for the spatial discretization of the viscous Burgers equation. We derive the numerical flux function from a local two-point boundary value problem (BVP), which results in a nonlinear equation that depends on the local boundary values and the diffusion constant. The flux scheme is consistent and stable (does not introduce any spurious oscillations), as demonstrated by the numerical results.
\end{abstract}

Keywords Numerical flux $\cdot$ Nonlinear local BVP $\cdot$ Viscous burgers equation

MSC (2010): $65 \mathrm{M} 08 \cdot 34 \mathrm{~B} 15$

\section{Introduction}

In this contribution we present a nonlinear flux approximation scheme for the spatial discretization of the viscous Burgers equation. The Burgers equation is an ideal test problem, as its spatial discretization can be carried over to the convective and viscous fluxes involved in the Navier-Stokes equations. The expression for the flux is derived from a local two-point BVP and is inspired by [5], where a local BVP is solved to

\footnotetext{
N. Kumar $(\varangle)$. J.H.M. ten Thije Boonkkamp · B. Koren

Department of Mathematics and Computer Science, Eindhoven University of Technology, PO Box 513, 5600MB Eindhoven, The Netherlands

e-mail: n.kumar@tue.nl

J.H.M. ten Thije Boonkkamp

e-mail: j.h.m.tenthijeboonkkamp@tue.nl

B. Koren

e-mail: b.koren@tue.nl

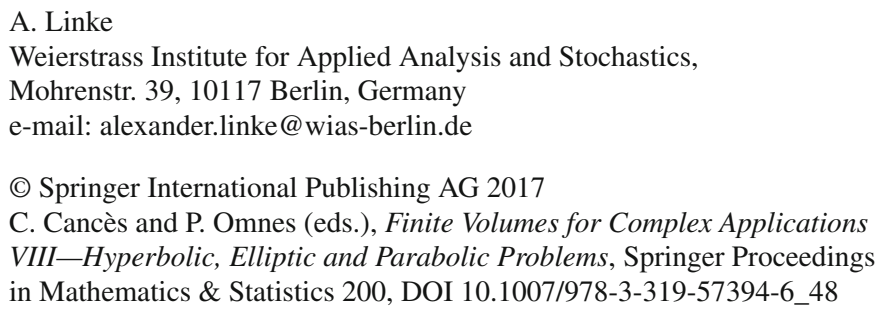


derive an integral representation of the flux for the convection-diffusion-reaction equation. The resulting numerical flux is expressed as a sum of a homogeneous part, which depends on the Péclet number (local balance of convection and diffusion) and an inhomogeneous part depending on the effects of the source term (associated with the reaction). Note that the homogeneous flux approximation is similar to the approximation methods described in $[1,3]$. In this contribution, we extend the homogeneous approximation to nonlinear problems.

In the vanishing viscosity limit, the viscous Burgers equation is a singularly perturbed problem. Moreover, the nonlinearity of the flux does not allow us to express the homogeneous flux as linear combination of the convective and the viscous part, which makes it cumbersome to have a consistent numerical flux. In this paper, we extend the local BVP method to nonlinear problems, such that the resulting numerical flux is consistent, i.e., reduces to the correct flux in the limit case. A discussion on nonlinear local two-point BVPs can be found in [2], where the authors show (i) the solvability of some auxiliary local nonlinear two-point BVPs, and (ii) the convergence of the discrete scheme to a weak solution of the continuous problem.

The paper is organized as follows: in Sect. 2 we formulate the local BVP for the flux approximation. Sect. 3 gives details of the derivation for the numerical fluxes. In Sect. 4 we compare the nonlinear scheme with the linearized homogeneous flux scheme described in [5] as well as with other standard methods. Sect. 5 gives the concluding remarks.

\section{Flux from Local Two-Point BVP}

Consider the one-dimensional viscous Burgers equation

$$
u_{t}+f\left(u, u_{x}\right)_{x}=0, \quad f\left(u, u_{x}\right):=\frac{1}{2} u^{2}-v u_{x},
$$

defined on $\Omega(\subset \mathbb{R}) \times(0, T)$, where $v(\geq 0)$ is the diffusion coefficient. The spatial discretization of the Burgers equation using a finite-volume method requires the approximation of the flux function $f\left(u, u_{x}\right)$ at each interface between two control volumes. The semi-discrete formulation of Eq. (1) is given by

$$
\Delta x \dot{u}_{j}+F_{j+1 / 2}-F_{j-1 / 2}=0, \quad \dot{u}:=u_{t},
$$

where $\left.F_{j+1 / 2} \approx f\left(u, u_{x}\right)\right|_{x=x_{j+1 / 2}}$, see Fig. 1 . The derivation of the flux $F_{j+1 / 2}$ is based on the following model BVP, in which we ignore the time dependence of the unknown:

$$
\begin{aligned}
& f_{x}=\left(\frac{1}{2} u^{2}-v u_{x}\right)_{x}=0, \quad x \in\left(x_{j}, x_{j+1}\right), \\
& u\left(x_{j}\right)=u_{j}=u_{L}, \quad u\left(x_{j+1}\right)=u_{j+1}=u_{R} .
\end{aligned}
$$


Fig. 1 Spatial discretization for the one-dimensional Burgers equation

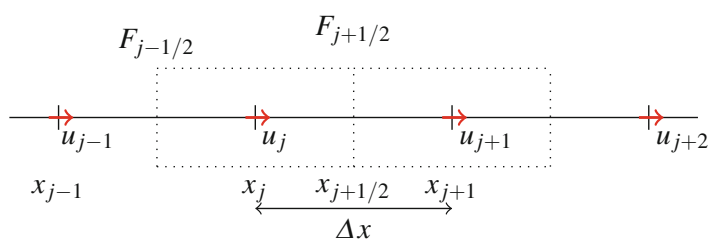

The solution of the nonlinear BVP (3) provides us the numerical flux function $\mathscr{F}\left(u_{L}, u_{R}, v / \Delta x\right)$, which is constant on the interval $\left(x_{j}, x_{j+1}\right)$. Thus, the numerical flux at the interface of the control volume $F_{j+1 / 2}=\mathscr{F}\left(u_{L}, u_{R}, v / \Delta x\right)$. Using the normalized coordinate $\sigma, \sigma \in[0,1]$ and the parameter $\varepsilon$, defined by

$$
\sigma:=\frac{x-x_{j}}{\Delta x}, \quad \varepsilon:=\frac{v}{\Delta x}
$$

the BVP (3) can be expressed as

$$
\begin{aligned}
& \left(\frac{1}{2} u^{2}-\varepsilon u_{\sigma}\right)_{\sigma}=0, \quad \sigma \in(0,1), \\
& u(0)=u_{L}, \quad u(1)=u_{R} .
\end{aligned}
$$

Further, it can be shown that the above BVP has a monotonic solution.

Lemma 1 The nonlinear local boundary value problem (4) has a strictly monotonic solution.

Proof Any solution $u$ of the problem can be represented as

$$
u(\sigma)=u_{L}+\left(u_{R}-u_{L}\right) \frac{\Lambda(\sigma)}{\Lambda(1)}, \quad u^{\prime}(\sigma)=\left(u_{R}-u_{L}\right) \frac{\lambda(\sigma)}{\Lambda(1)}, \quad()^{\prime}:=\frac{\mathrm{d}}{\mathrm{d} \sigma}
$$

for $\sigma \in[0,1]$, where the functions $\lambda, \Lambda:[0,1] \rightarrow \mathbb{R}$ are given by

$$
\lambda(\sigma):=\exp \left(\frac{1}{\varepsilon} \int_{0}^{\sigma} u(\eta) \mathrm{d} \eta\right) \text { and } \Lambda(\sigma):=\int_{0}^{\sigma} \lambda(\xi) \mathrm{d} \xi
$$

For $u_{L}>u_{R}, u^{\prime}(\sigma)<0$ causing $u(\sigma)$ to be a monotonically decreasing function. Similarly, if $u_{L}<u_{R}$, then $u^{\prime}(\sigma)>0$ and $u$ is monotonically increasing.

\section{The Numerical Flux Function}

We now derive expressions for the numerical flux function using the BVP (4). As a consequence of Lemma 1 we consider the cases: $u_{L}>u_{R}$ and $u_{L}<u_{R}$. 


\subsection{The Case $u_{L}>U_{R}$}

The solution of the BVP (4) in this case results in a (strictly) decreasing function, i.e., $u_{\sigma}<0$. Using the left boundary condition $u(0)=u_{L}$, we get that the numerical flux at the interface, $F_{j+1 / 2}$, is given by

$$
F_{j+1 / 2}=f(0)=\frac{1}{2} u_{L}^{2}-\varepsilon u_{\sigma}(0) .
$$

Alternatively, the flux can be determined using the right boundary condition

$$
F_{j+1 / 2}=\frac{1}{2} u_{R}^{2}-\varepsilon u_{\sigma}(1)
$$

Since $u_{\sigma}<0$, we conclude that $F_{j+1 / 2}>0$, therefore there exists a $c \in \mathbb{R}$, such that

$$
F_{j+1 / 2}=\frac{1}{2} u^{2}-\varepsilon u_{\sigma}=\frac{1}{2} u_{L}^{2}-\varepsilon u_{\sigma}(0)=\frac{1}{2} u_{R}^{2}-\varepsilon u_{\sigma}(1)=\frac{1}{2} c^{2},
$$

with $|c| \geq \max \left(\left|u_{L}\right|,\left|u_{R}\right|\right)$. The above relation gives us the first-order differential equation

$$
\frac{\mathrm{d} u}{\mathrm{~d} \sigma}=\frac{1}{2 \varepsilon}\left(u^{2}-c^{2}\right), \quad \sigma \in(0,1),
$$

which needs to satisfy both $u(0)=u_{L}$ and $u(1)=u_{R}$. Integrating the differential equation and connecting the left boundary condition with the right boundary condition results in the following nonlinear equation for the unknown $c$ with parameters $u_{L}, u_{R}$ and $\varepsilon$

$$
H^{+}(c):=\log \left|\frac{\left(u_{L}+c\right)\left(u_{R}-c\right)}{\left(u_{L}-c\right)\left(u_{R}+c\right)}\right|-\frac{c}{\varepsilon}=0 .
$$

Thus, $F_{j+1 / 2}$ is given by the non-trivial roots of the function $H^{+}(c)$, which is an odd function. We restrict ourselves to $c>0$. Note that the nonlinear equation (9) can also be expressed as

$$
e^{-c / 2 \varepsilon}\left|\left(u_{L}+c\right)\left(u_{R}-c\right)\right|-e^{c / 2 \varepsilon}\left|\left(u_{L}-c\right)\left(u_{R}+c\right)\right|=0 .
$$

Let $s=\left(u_{L}+u_{R}\right) / 2$, then for $s \geq 0$, we get that $u_{L} \geq\left|u_{R}\right|$ and the non-trivial solution of Eq. (10) satisfies $c \geq u_{L} \geq\left|u_{R}\right|$. In the inviscid limit $\varepsilon \rightarrow 0$, for $s \geq 0$ Eq. (10) reduces to

$$
e^{c / 2 \varepsilon}\left(c-u_{L}\right)\left(c+u_{R}\right)=0 \Rightarrow c=u_{L} .
$$

Similarly for $s<0$, we have $u_{R}<0$, implying $c \geq-u_{R} \geq\left|u_{L}\right|$ and the limit case solution is then given by $c=-u_{R}(>0)$. Thus, the numerical flux in the inviscid limit is given by 


$$
F_{j+1 / 2}= \begin{cases}\frac{1}{2} u_{L}^{2}, & \text { if } s \geq 0 \\ \frac{1}{2} u_{R}^{2}, & \text { if } s<0\end{cases}
$$

which is actually the Godunov flux for the inviscid Burgers equation. Moreover, if $u_{L}=u_{R}=u$, then $u_{\sigma}=0$ and the numerical flux is given by $F_{j+1 / 2}=\mathscr{F}(u, u)=$ $\frac{1}{2} u^{2}=f(u)$, for constant $u$. Hence the numerical flux function $\mathscr{F}$ is consistent with the continuous flux function $f$.

\subsection{The Case $u_{L}<U_{R}$}

From Lemma 1 we conclude that $u_{\sigma}>0$ for $u_{L}<u_{R}$. Thus $F_{j+1 / 2}=u^{2} / 2-\varepsilon u_{\sigma}$ is positive if $\varepsilon u_{\sigma}<u^{2} / 2$ and negative if $\varepsilon u_{\sigma}>u^{2} / 2$. Therefore, we split the derivation of the numerical flux into two cases, depending on the sign of the flux.

\section{Case 1: Positive flux}

If the flux is positive, then the numerical flux is evaluated as for the case $u_{L}>u_{R}$ and is given by roots of the function $H^{+}(c)$, defined in Eq. (9), with $c \in(0, M)$, $M:=\min \left(\left|u_{L}\right|,\left|u_{R}\right|\right)$.

\section{Case 2: Negative flux}

If the flux is negative, then there exists a $c \in \mathbb{R}$, such that

$$
F_{j+1 / 2}=\frac{1}{2} u^{2}-\varepsilon u_{\sigma}=-\frac{1}{2} c^{2} .
$$

This relation gives rise to the first-order differential equation

$$
\frac{\mathrm{d} u}{\mathrm{~d} \sigma}=\frac{1}{2 \varepsilon}\left(u^{2}+c^{2}\right), \quad \sigma \in(0,1),
$$

with the boundary conditions (4b). Integrating the first-order differential equation and connecting the left boundary condition with the right boundary condition gives us another nonlinear equation for $c$, i.e.,

$$
H^{-}(c):=\arctan \left(\frac{u_{R}}{c}\right)-\arctan \left(\frac{u_{L}}{c}\right)-\frac{c}{2 \varepsilon}=0 .
$$

As before, the numerical value of $F_{j+1 / 2}=-c^{2} / 2$ is given by the non-trivial roots of the function $H^{-}(c)$. We restrict ourselves to the case $0<u_{L}<u_{R}$.

We now formulate the conditions for which $H^{+}(c)$ and $H^{-}(c)$ have non-trivial roots.

Lemma 2 For $0<u_{L}<u_{R}$, if the inequality

$$
\frac{1}{u_{L}}-\frac{1}{u_{R}}>\frac{1}{2 \varepsilon},
$$


holds then $\mathrm{H}^{-}(\mathrm{c})$ has a non-trivial solution, otherwise $\mathrm{H}^{+}(\mathrm{c})$ has a non-trivial solution.

Proof Let $\alpha_{-}(c):=\arctan \left(u_{R} / c\right)-\arctan \left(u_{L} / c\right)$ and $\beta_{-}(c):=c / 2 \varepsilon$, such that $H^{-}(c):=\alpha_{-}(c)-\beta_{-}(c)$. Using $\arctan (1 / z)=-\arctan (z)+\pi \operatorname{sgn}(z) / 2, \alpha_{-}(c)$ can be expressed as

$$
\alpha_{-}(c)=\arctan \left(\frac{c}{u_{L}}\right)-\arctan \left(\frac{c}{u_{R}}\right)+\frac{\pi}{2}\left(\operatorname{sgn}\left(u_{R}\right)-\operatorname{sgn}\left(u_{L}\right)\right) .
$$

Using the fact that $\alpha_{-}(c)$ is an odd function we restrict ourselves to the case $c>0$. For $0<u_{L}<u_{R}, \alpha_{-}(c)$ has a maximum at $c=\sqrt{u_{L} u_{R}}\left(<u_{R}\right)$. Clearly $H^{-}(c)$ has a non-trivial root whenever $\alpha_{-}(c)=\beta_{-}(c)$, i.e., the two functions intersect for $c>0$, which is possible only if $\alpha_{-}^{\prime}(0)>\beta_{-}^{\prime}(0)$, or,

$$
\alpha_{-}^{\prime}(0)=\frac{1}{u_{L}}-\frac{1}{u_{R}}>\beta_{-}^{\prime}(0)=\frac{1}{2 \varepsilon}, \quad(.)^{\prime}=\frac{\mathrm{d}}{\mathrm{d} c} .
$$

Thus, if the above condition holds then $H^{-}(c)$ has a non-trivial solution, which satisfies $\sqrt{u_{L} u_{R}}<c<u_{R}$.

Next, we investigate the condition under which $H^{+}(c)$ has a non-trivial root. Let

$$
z(c):=\frac{\left(u_{R}-u_{L}\right) c}{u_{L} u_{R}-c^{2}},
$$

such that $z \in[0,1]$ with $z(0)=0, z\left(u_{L}\right)=1$. Clearly, for $c \in\left(0, u_{L}\right)$ we have $z(c)>$ 0 . Using $z(c)$ we can rewrite Eq. (9) as

$$
H^{+}(c)=\log \left(\frac{1+z(c)}{1-z(c)}\right)-\frac{c}{\varepsilon}=2 \operatorname{Artanh}(z(c))-\frac{c}{\varepsilon}, \quad c \in\left(0, u_{L}\right) .
$$

Further, let $\alpha_{+}(c):=2 \operatorname{Artanh}(z(c))$ and $\beta_{+}(c):=c / \varepsilon$, such that $H^{+}(c)=\alpha_{+}(c)-$ $\beta_{+}(c)$. For $c \in\left(0, u_{L}\right), \alpha_{+}(c)$ is an increasing function, thus $H^{+}(c)$ has a non-trivial root only if $\alpha_{+}^{\prime}(0)<\beta_{+}^{\prime}(0)$. The derivative $\alpha_{+}^{\prime}(c)$ is given by

$$
\alpha_{+}^{\prime}(c)=2\left(u_{R}-u_{L}\right) \frac{1}{1-z^{2}(c)} \frac{u_{L} u_{R}+c^{2}}{\left(u_{L} u_{R}-c^{2}\right)^{2}} .
$$

The condition $\alpha_{+}^{\prime}(0)<\beta_{1}^{\prime}(0)$ translates to

$$
\frac{1}{u_{L}}-\frac{1}{u_{R}}<\frac{1}{2 \varepsilon}
$$

Lastly, integrating the differential equation (8) with $c=0$ gives us the condition for zero-flux: $1 / u_{L}-1 / u_{R}=1 / 2 \varepsilon$, which is in agreement with the above criteria for positive or negative flux. 
Fig. $2 H^{+}(c)$ (a) and $H^{-}(c)$ (b), for $u_{L}=0.75$, $u_{R}=1.0$ and $\varepsilon=0.1$
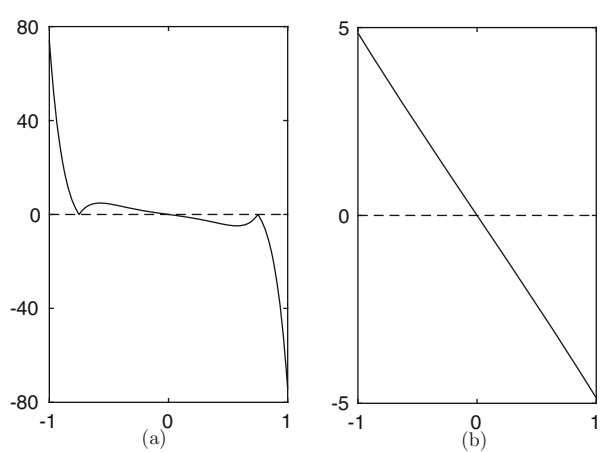

Fig. $3 H^{+}(c)$ a and $H^{-}(c)$ b, for $u_{L}=1, u_{R}=10$ and $\varepsilon=1$
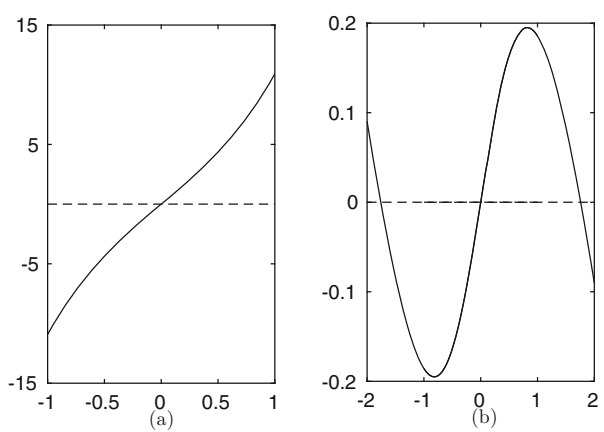

Figure 2 shows the plots of the functions $H^{+}(c)$ and $H^{-}(c)$, for $u_{L}=0.75, u_{R}=1$ and $\varepsilon=0.1$ not satisfying (14). Hence, $H^{-}(c)$ does not have a non-trivial root, unlike $H^{+}(c)$ which has a non-trivial root at $c=0.749\left(\approx u_{R}\right)$. In Fig. 3, for $u_{L}=1$, $u_{R}=10$ and $\varepsilon=1$, condition (14) is satisfied. Thus, $H^{+}(c)$ does not have a nontrivial root, whereas $H^{-}(c)$ has a non-trivial root, at $c=1.7597$.

\section{Numerical Results}

We compare the proposed nonlinear local BVP scheme with the upwind scheme and the homogeneous flux scheme described in $[4,5]$. In the homogeneous flux scheme, the numerical flux $F_{j+1 / 2}^{\mathrm{lin}}$ is derived from a linearized homogeneous local two-point $\mathrm{BVP}$ and is given by

$$
F_{j+1 / 2}^{\operatorname{lin}}=\varepsilon\left(B(-P) u_{L}-B(P) u_{R}\right)
$$

where $B(z):=z /\left(e^{z}-1\right)$ is the Bernoulli function and $P:=U_{j+1 / 2} / 2 \varepsilon$, is the grid Péclet number. The interface velocity $U_{j+1 / 2}=\left(u_{L}+u_{R}\right) / 2$ is given by the central approximation. The availability of an analytical solution to the viscous Burgers 
equation defined on $(0,1) \times(0, T), T \in(0,1]$ provides us a reference solution to compare the schemes:

$$
u^{\mathrm{ref}}(x, t)=1+\frac{1}{2} \tanh \left(\frac{1}{4 v}\left(x-0.1-\frac{1}{2} t\right)\right) .
$$

We use the explicit fourth-order Runge-Kutta scheme for the temporal discretization with $\Delta t=10^{-3}$. For this test case, we have $0<u_{R}<u_{L}$ throughout the computational domain, and the numerical flux is given by the roots of $H^{+}(c)$. The Newton solver converges in 2-8 iteration steps (depending on the tolerance, ranging from $10^{-3}$ to $10^{-8}$ ), for a good initial guess. A fairly accurate initial guess can be derived using the bounds on the derivative $u_{\sigma}$, that can be obtained using Lemma 1 . Figure 4 shows the convergence of the error $\mathbf{e}_{\mathbf{u}}:=\left|\mathbf{u}-\mathbf{u}^{\text {ref }}\right|_{1}$ for $v=10^{-3}$ over a family of uniform grids. Grid refinement (for fixed $v$ ) causes $\varepsilon$ to increase (for the test case, $\left.\varepsilon=2^{i} \times 10^{-2}, i=1,2, \ldots, 6\right)$. Moreover, it is observed that the root-finder converges faster for higher values of $\varepsilon(\approx 1)$ than for smaller values of $\varepsilon$. On coarse grids all three schemes exhibit first-order accuracy, with the local BVP schemes being slightly more accurate than the upwind scheme. However, on grid refinement (increasing $\varepsilon$ ) the nonlinear BVP scheme is found to be more accurate compared to the upwind and the linearized local BVP scheme (Fig. 4). Further, Richardson extrapolation shows that for finer grids the nonlinear local BVP scheme exhibits second-order convergence.

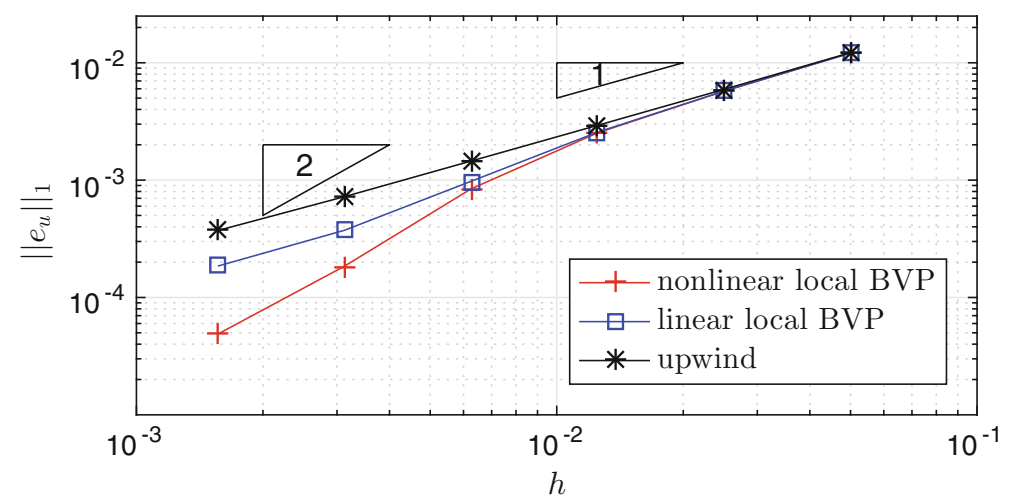

Fig. 4 Convergence of the 1-norm of the error $e_{u}$ for $v=10^{-3}$ for the proposed nonlinear local BVP scheme, homogeneous linear local BVP scheme and the upwind scheme for a family of grids $\left(\Delta x=0.1 \times 2^{-i} ; i=1,2,3,4,5,6\right)$ 


\section{Conclusion}

In this paper, we have presented a flux approximation scheme for the viscous Burgers equation, in which the numerical flux function is given by the solution of a local nonlinear two-point BVP, resulting in a locally exact approximation that corresponds with the nonlinearity of the flux function. The resulting numerical flux is shown to be consistent with the Godunov method in the inviscid limit and is more accurate than the linearized homogeneous approximation scheme in $[4,5]$.

In the future, we plan to extend the scheme by including source terms and also the time derivative into the local BVP, and by then solving the inhomogeneous BVP, to get the nonlinear complete-flux scheme.

Acknowledgements This work is part of the Industrial Partnership Programme (IPP) Computational Sciences for Energy Research of the Foundation for Fundamental Research on Matter (FOM), which is part of the Netherlands Organization for Scientific Research (NWO).

\section{References}

1. Allen, D.N.G., Southwell, R.V.: Relaxation methods applied to determine the motion, in two dimensions, of a viscous fluid past a fixed cylinder. Q. J. Mech. Appl. Math. 8, 129-145 (1955)

2. Eymard, R., Fuhrmann, J., Gärtner, K.: A finite volume scheme for nonlinear parabolic equations derived from one-dimensional local Dirichlet problems. Numeri. Math. 102, 463-495 (2006)

3. Il'in, A.M.: Differencing scheme for a differential equation with a small parameter affecting the highest derivative. Math. Notes Acad. Sci. USSR 6, 596-602 (1969)

4. Kumar, N., ten Thije Boonkkamp, J., Koren, B.: Flux approximation cheme for the incompressible Navier-Stokes equations using local boundary value problems. In: Lecture Notes in Computational Science and Engineering, vol. 112, pp. 43-51. Springer, Heidelberg (2016)

5. ten Thije Boonkkamp, J.H.M., Anthonissen, M.J.H.: The finite volume-complete flux scheme for advection-diffusion-reaction equations. J. Sci. Comput. 46, 47-70 (2011) 